DESY 08-037

ISSN 0418-9833

arXiv:0811.1364 [hep-ph]

November 2008

\title{
Two-loop electroweak fermionic corrections to $\sin ^{2} \theta_{\mathrm{eff}}^{b \bar{b}}$
}

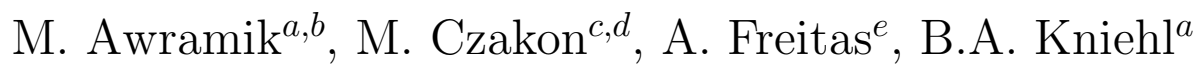 \\ a II. Institut für Theoretische Physik, Universität Hamburg, \\ Luruper Chaussee 149, 22761 Hamburg, Germany \\ ${ }^{b}$ Institute of Nuclear Physics PAN, \\ ul. Radzikowskiego 152, 31342 Kraków, Poland \\ c Institut für Theoretische Physik und Astrophysik, Universität Würzburg, \\ Am Hubland, 97074 Würzburg, Germany \\ 'Institute of Nuclear Physics, NCSR "DEMOKRITOS", \\ 15310 Athens, Greece \\ e Department of Physics 8 Astronomy, University of Pittsburgh, \\ Pittsburgh, PA 15260, USA
}

\begin{abstract}
We present the first calculation of the two-loop electroweak fermionic correction to the flavour-dependent effective weak-mixing angle for bottom quarks, $\sin ^{2} \theta_{\mathrm{eff}}^{b \bar{b}}$. For the evaluation of the missing two-loop vertex diagrams, two methods are employed, one based on a semi-numerical Bernstein-Tkachov algorithm and the second on asymptotic expansions in the large top-quark mass. A third method based on dispersion relations is used for checking the basic loop integrals. We find that for small Higgs-boson mass values, $M_{H} \propto 100 \mathrm{GeV}$, the correction is sizable, of order $\mathcal{O}\left(10^{-4}\right)$.
\end{abstract}

Keywords: Electroweak radiative corrections, effective weak-mixing angle, BernsteinTkachov algorithm

PACS: 12.15.Lk, 13.38.Dg, 13.66.Jn, 14.70.Hp 


\section{Introduction}

Experiments at LEP, SLC and Tevatron have provided a large number of high-precision data, which, being supplemented by detailed studies of higher-order corrections, allow to probe the Standard Model at the loop level and subsequently to predict the mass of the Higgs boson. In this context, the leptonic effective weak-mixing angle, $\sin ^{2} \theta_{\text {eff }}^{\text {lept }}$, plays the most crucial role. It can be defined through the effective vector and axial-vector couplings, $g_{V}^{l}$ and $g_{A}^{l}$, of the $Z$ boson to leptons $(l)$ at the $Z$-boson pole,

$$
\sin ^{2} \theta_{\mathrm{eff}}^{\mathrm{lept}}=\frac{1}{4}\left(1+\operatorname{Re} \frac{g_{V}^{l}}{g_{A}^{l}}\right) .
$$

The effective weak-mixing angle can be related to the on-shell Weinberg angle, $\sin ^{2} \theta_{w}$, as

$$
\sin ^{2} \theta_{\mathrm{eff}}^{\text {lept }}=\sin ^{2} \theta_{w} \kappa,
$$

where $\sin ^{2} \theta_{w}=1-M_{W}^{2} / M_{Z}^{2}$ and $\kappa=1+\Delta \kappa$. At tree level, $\Delta \kappa=0$ and $\sin ^{2} \theta_{\text {eff }}^{\text {lept }}=\sin ^{2} \theta_{w}$. The form factor $\Delta \kappa$ incorporates the higher-order loop corrections. Usually, the $W$-boson mass, $M_{W}$, is not treated as an input parameter but it is calculated from the Fermi constant, $G_{\mu}$, which is precisely known from the muon lifetime. The relation between $M_{W}$ and $G_{\mu}$ can be cast in the form

$$
M_{W}^{2}\left(1-\frac{M_{W}^{2}}{M_{Z}^{2}}\right)=\frac{\pi \alpha}{\sqrt{2} G_{\mu}}(1+\Delta r),
$$

where the quantity $\Delta r[1]$ contains all higher-order corrections. The presently most accurate calculation of the $W$-boson mass includes full two-loop and leading higher-order corrections [2]. On the other hand, the quantity $\kappa$ in Eq. (2) incorporates all corrections to the form factors of the $Z l \bar{l}$ vertex. Recently, the calculation of the two-loop electroweak corrections has been completed [3-7]. The uncertainty on $\sin ^{2} \theta_{\text {eff }}^{\text {lept }}$ due to unknown higher orders has been estimated to be 0.000047 , which is substantially smaller than the error of the current experimental value $\sin ^{2} \theta_{\text {eff }}^{\text {lept }}=0.23153 \pm 0.00016$ [8], but still larger than the expected precision, $1.3 \times 10^{-5}$, of a future high-luminosity linear collider running at the $Z$-boson pole [9].

The experimental value for $\sin ^{2} \theta_{\text {eff }}^{\text {lept }}$ is determined from six asymmetry measurements, $\mathcal{A}_{F B}^{0, l}, \mathcal{A}_{l}\left(P_{\tau}\right), \mathcal{A}_{l}(\mathrm{SLD}), \mathcal{A}_{F B}^{0, b}, \mathcal{A}_{F B}^{0, c}$, and $\mathcal{Q}_{F B}^{\text {had }}$. Of those, the average leptonic and hadronic measurements differ by 3.2 standard deviations, which is one of the largest discrepancies within the Standard Model. The main impact stems from two measurements, the leftright asymmetry with a polarised electron beam at SLD, $\mathcal{A}_{L R}^{0}$, and the forward-backward asymmetry for bottom quarks at LEP, $\mathcal{A}_{F B}^{0, b}$. On the experimental side, the only possible source of this discrepancy are uncertainties in external input parameters, in particular parameters describing the production and decay of heavy-flavoured hadrons; see Section 5 of Ref. [8] for a discussion. However, the interpretation of the asymmetry measurements in terms of $\sin ^{2} \theta_{\text {eff }}^{\text {lept }}$ requires also some theoretical input. The leptonic asymmetries depend 
on lepton couplings only and can be translated straightforwardly into the leptonic effective weak-mixing angle, with small corrections due to $s$ - and $t$-channel photon exchange. By contrast, the hadronic observables, $\mathcal{A}_{F B}^{0, c}, \mathcal{A}_{F B}^{0, b}$ and $\mathcal{Q}_{F B}^{\text {had }}$, depend on the quark couplings, $g_{V, A}^{q}$. These couplings are associated with a flavour-dependent hadronic effective weakmixing angle, $\sin ^{2} \theta_{\mathrm{eff}}^{q \bar{q}}$,

$$
\sin ^{2} \theta_{\mathrm{eff}}^{q \bar{q}}=\frac{1}{4\left|Q_{q}\right|}\left(1+\operatorname{Re} \frac{g_{V}^{q}}{g_{A}^{q}}\right), \quad q=d, u, s, c, b .
$$

The forward-backward pole asymmetry of a quark $q, \mathcal{A}_{F B}^{0, q}$, is related to the effective couplings, $g_{V}^{f}$ and $g_{A}^{f}$, and the effective weak-mixing angle, $\sin ^{2} \theta_{\mathrm{eff}}^{q \bar{q}}$, by ${ }^{1}$

$$
\mathcal{A}_{F B}^{0, q}=\frac{3}{4} \mathcal{A}_{e} \mathcal{A}_{q}
$$

with

$$
\mathcal{A}_{f}=\frac{2 g_{V}^{f} g_{A}^{f}}{\left(g_{V}^{f}\right)^{2}+\left(g_{A}^{f}\right)^{2}}=\frac{1-4\left|Q_{f}\right| \sin ^{2} \theta_{\mathrm{eff}}^{f \bar{f}}}{1-4\left|Q_{f}\right| \sin ^{2} \theta_{\mathrm{eff}}^{f \bar{f}}+8 Q_{f}^{2} \sin ^{4} \theta_{\mathrm{eff}}^{f \bar{f}}} .
$$

At tree level, $\sin ^{2} \theta_{\text {eff }}^{q \bar{q}}$ and $\sin ^{2} \theta_{\text {eff }}^{\text {lept }}$ are identical, but the relations between these quantities receive sizable radiative corrections that need to be included in the analysis. Note that, due to the small electric charge of the bottom quark, $Q_{b}=-1 / 3$, the parameter $\mathcal{A}_{b}$ is close to 1 , and $\mathcal{A}_{F B}^{0, b}$ is only weakly sensitive to $\sin ^{2} \theta_{\text {eff }}^{b \bar{b}}$. Therefore, it seems unlikely that the discrepancy between $\mathcal{A}_{L R}^{0}$ and $\mathcal{A}_{F B}^{0, b}$ could be explained by radiative corrections. Nevertheless, the theoretical prediction for $\sin ^{2} \theta_{\text {eff }}^{b \bar{b}}$ enters in the Standard-Model fits through several observables, so that a precise prediction of this quantity is important for a robust analysis.

For all fermions except bottom quarks, the known radiative corrections to $\sin ^{2} \theta_{\text {eff }}^{f \bar{f}}$ include at least two-loop fermionic electroweak contributions and some leading higher-order corrections; see Ref. [7] for details. However, for the $Z b \bar{b}$ vertex only one-loop corrections, leading two-loop corrections for large values of the top-quark mass of $\mathcal{O}\left(\alpha^{2} m_{t}^{4}\right)$, and two- and three-loop QCD corrections have been calculated [10] and included in the ZFITTER program [11] (see also the new program GFITTER [12]), which is widely used for global Standard-Model fits. The remaining two-loop electroweak corrections beyond the $\mathcal{O}\left(\alpha^{2} m_{t}^{4}\right)$ contributions are still unknown, although they are expected to be larger than the $\mathcal{O}\left(\alpha^{2} m_{t}^{4}\right)$ term, based on experience from $\sin ^{2} \theta_{\text {eff }}^{\text {lept }}$. As a result, the present treatment of higher-order electroweak corrections leads to inconsistencies, for example in $\mathcal{A}_{F B}^{0, b}$, since the corrections to $\sin ^{2} \theta_{\text {eff }}^{\text {lept }}$ and $\mathcal{A}_{e}$ include two-loop and leading three-loop corrections that are absent for $\sin ^{2} \theta_{\text {eff }}^{b \bar{b}}$ and $\mathcal{A}_{b}$ (see recent discussion in Ref. [13]).

In this paper, the part of the missing two-loop corrections to $\sin ^{2} \theta_{\text {eff }}^{b \bar{b}}$ with closed fermion loops is presented. We begin by explaining the techniques employed for the

\footnotetext{
${ }^{1}$ Owing to the non-zero bottom-quark mass, the $Z b \bar{b}$ vertex also has a scalar part, besides the vector and axial-vector parts. We checked explicitly that the contribution of this scalar form factor to $\mathcal{A}_{F B}^{0, b}$ is more than a factor 1000 smaller than the current experimental uncertainty and thus truly negligible.
} 
calculation in the next section. In Section 3, numerical results for $\sin ^{2} \theta_{\text {eff }}^{b \bar{b}}$ are given before the summary in Section 4.

\section{Outline of the calculation}

\subsection{General approach}

We work in the Standard Model and adopt the on-shell renormalisation scheme, which relates the renormalised masses and couplings to physical observables. Details on the renormalisation scheme and explicit expressions for the relevant counterterms can be found in Refs. [7, 14]. For the loop integrations, we employ dimensional regularisation. The problem of $\gamma_{5}$ matrices in two-loop vertex diagrams with fermion triangle sub-loops is treated in the same way as in Refs. $[3,4,7]$, by evaluating the finite non-anticommutative contribution from $\gamma_{5}$ to the vertex diagrams in four dimensions. Most aspects connected with the calculation of the effective weak-mixing angle for the $Z b \bar{b}$ vertex are the same as for the leptonic effective weak-mixing angle and are discussed in detail in Ref. [7].

The contributions for the two-loop renormalisation terms are identical to the case of $\sin ^{2} \theta_{\text {eff }}^{\text {lept }}$, with the exception of the two-loop bottom-quark wave-function counterterm, which involves new self-energy diagrams with internal top-quark propagators; the first terms of this quantity are given in Ref. [15]. For the two-loop $Z b \bar{b}$ vertex corrections, on the other hand, a number of new three-point diagrams need to be computed. In general, electroweak two-loop corrections can be divided into two groups, which are separately finite and gauge invariant: fermionic corrections (with at least one closed fermion loop) and bosonic corrections (without any closed fermion loops). In this article, we focus on the fermionic diagrams as a first step.

For the purpose of this calculation, all light-quark masses are neglected in the twoloop diagrams, including the bottom-quark mass. As a result, for many diagrams, known results from the $\sin ^{2} \theta_{\text {eff }}^{\text {lept }}$ calculation can be used $[3,4,7]$. The loop integrals for diagrams with closed massless-fermion loops are given in analytical form, while large-mass expansions were employed for diagrams with top quarks in the loops.

However, the two-loop corrections to $\sin ^{2} \theta_{\text {eff }}^{b \bar{b}}$ include a new group of integrals that were not covered in previous calculations of $\sin ^{2} \theta_{\text {eff }}^{\text {lept }}$, stemming from diagrams with internal $W$-boson and top-quark propagators; see Fig. 1 . The computation of these diagrams will be discussed in detail in the following subsections. The two-loop diagrams are computed with several independent methods, so that cross checks can be performed. The first method, based on the observation that all new diagrams in Fig. 1 include internal topquark propagators, uses asymptotic expansions for large top-quark mass. This method was already employed successfully for the calculation of $\sin ^{2} \theta_{\text {eff }}^{\text {lept }}$ [7]. For references on the subject, we refer the reader to Ref. [16].

Secondly, we develop a code for the evaluation of Feynman diagrams with a seminumerical method, based on the Bernstein-Tkachov (BT) method of Ref. [17]. This method had already been used previously for one-loop problems [18]. In a recent series of 
(A)

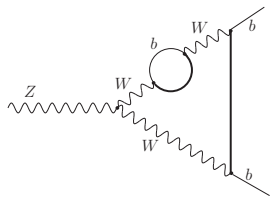

(C)

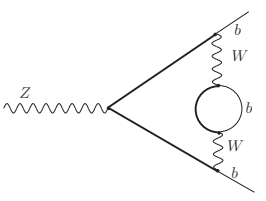

(E)

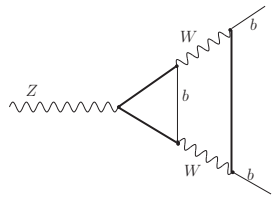

(B)

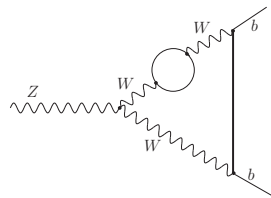

(D)

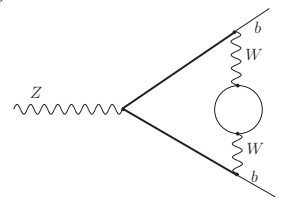

Figure 1: Set of Feynman diagrams required for the calculation of the fermionic two-loop corrections to the $Z b \bar{b}$ vertex, but absent in the $\sin ^{2} \theta_{\text {eff }}^{\text {lept }}$ case. Thick solid lines denote top-quark propagators, while thin lines represent light fermions.

papers [19], it was extended to general two-loop vertices, and some applications to twoloop problems are already known: the leptonic effective weak-mixing angle was presented in Ref. [5] and corrections to the $H \rightarrow \gamma \gamma$ decay width in Ref. [20].

Finally, we use another semi-numerical method based on dispersion relations [21], which was also used previously for $\sin ^{2} \theta_{\text {eff }}^{\text {lept }}[7]$. This method allows us to evaluate all self-energy diagrams, the vertex diagrams in Figs. 1(A)-(D), as well as the scalar integrals with the topology of Figs. 1(E)- $(\mathrm{G})$. However, due to problems with the complex tensor structure, the complete diagrams in Figs. $1(\mathrm{E})-(\mathrm{G})$ cannot be checked with this technique.

In the next subsections, we explain the applications of these methods for our purposes and present a comparison between them.

\section{$2.2 \quad$ Asymptotic expansions}

We perform an expansion in a parameter $x$, where

$$
x=\frac{M_{Z}^{2}}{m_{t}^{2}} \sim \frac{1}{4} .
$$

For any two-loop problem, there are four regions to consider. Let $k_{1}$ and $k_{2}$ represent the internal momenta in the loops and $p$ stand for any external momentum, while $m$ 
generically denotes all masses that are small compared to $m_{t}, m<m_{t}$. In our case, $m=M_{W}, M_{Z}$. Then the four regions can be identified as follows:

1) $k_{1} \sim m_{t}$ and $k_{2} \sim m_{t} \quad$ (expansions in small parameters: $p$ and $m$ )

2) $k_{1} \sim m$ and $k_{2} \sim m_{t} \quad$ (expansions in small parameters: $p, k_{1}$ and $m$ )

3) $k_{1} \sim m_{t}$ and $k_{2} \sim m \quad$ (expansions in small parameters: $p, k_{2}$ and $m$ )

4) $k_{1} \sim m$ and $k_{2} \sim m \quad$ (expansions in small parameters: $p, k_{1}, k_{2}$ and $m$ )

This method allows us to represent two-loop vertex diagrams by a sum of simpler integrals, namely two-loop propagator and vacuum integrals, plus one-loop integrals. However, higher orders in the expansion lead to higher powers of propagator denominators in these integrals. This is not a problem for one-loop or vacuum integrals, as analytic relations are well known; for relations and references, see, for example, Ref. [16]. For two-loop propagator integrals, we employ the Laporta algorithm, as proposed in Ref. [22]. This algorithm allows us to automatically reduce complicated multi-loop integrals with nontrivial numerators to a smaller set of master integrals with unit numerators. In addition to the well-known integration by parts relations [23], Lorentz identities [24] can be used for faster performance. In our approach, the Laporta integral reduction is accomplished with the help of the program IDSOLver [4,25].

As already observed for the two-loop vertex diagrams in the leptonic case [7], this expansion has a fast convergence behaviour. After performing the expansion down to fifth order, $\mathcal{O}\left(x^{5}\right)$, a precision of $10^{-5}$ in the final result for the two-loop part of $\sin ^{2} \theta_{\text {eff }}^{b \bar{b}}$ is obtained.

\subsection{Semi-numerical integration based on the BT algorithm}

Any Feynman diagram can be described by an integral

$$
\int_{\mathcal{S}} \mathrm{d} \mathbf{x} \mathcal{Q}(\mathbf{x}) \prod_{i} \mathcal{V}_{i}^{\mu_{i}}(\mathbf{x})
$$

where $\mathcal{Q}(\mathbf{x})$ and $\mathcal{V}_{i}(\mathbf{x})$ are polynomials of $\mathbf{x}, \mathcal{Q}$ is the numerator of a Feynman integral, $\mathcal{V}_{i}$ is a denominator of the Feynman integral with the power $\mu_{i}$, which depends on $\varepsilon$, $\mathbf{x}=\left(x_{1}, \ldots, x_{n}\right)$ represents the $n$-dimensional vector space of Feynman parameters, and $\mathcal{S}$ is the integration region defined by

$$
\int_{\mathcal{S}} \mathrm{d} \mathbf{x}=\int_{0}^{1} \mathrm{~d} x_{1} \int_{0}^{1-x_{1}} \mathrm{~d} x_{2} \ldots \int_{0}^{1-\sum_{i=1}^{n-1} x_{i}} \mathrm{~d} x_{n} .
$$

Tkachov proved the existence of an algorithm [17], later called BT algorithm, that can transform a Feynman integral of the type in Eq. (8) into a form with better arrangement of divergences, which is consequently more suitable for numerical integration. However, until now, compact-form solutions of this algorithm are only known at the one-loop order, which will be described in the following.

For one-loop cases, $\mathcal{V}(\mathbf{x})$ is a quadratic polynomial of $\mathbf{x}$ of the form

$$
\mathcal{V}(\mathbf{x})=\mathbf{x}^{T} \mathcal{W} \mathbf{x}+2 \mathbf{R}^{T} \mathbf{x}+Z
$$


where $\mathcal{W}$ is a $n \times n$ matrix, $\mathbf{R}$ is a $n$-dimensional vector and $Z$ is a scalar number. Then one can show that the following relation is fulfilled:

$$
\mathcal{V}^{\mu}=\frac{1}{\Delta}\left(1-\frac{(\mathbf{x}+\mathbf{A}) \boldsymbol{\partial}}{2(\mu+1)}\right) \mathcal{V}^{\mu+1}
$$

where $\Delta=\left(Z-\mathbf{R}^{T} \mathcal{W}^{-1} \mathbf{R}\right)$ and $\mathbf{A}=\mathbf{R}^{T} \mathcal{W}^{-1}$. By application of this one-loop BT relation, supplemented by integration-by-parts identities, the power of the polynomial $\mathcal{V}(\mathbf{x})$ of the Feynman integral is raised. For example, for the one-loop three-point function in three dimensions, one finds

$$
\begin{aligned}
& \int_{0}^{1} \mathrm{~d} x_{1} \int_{0}^{1-x_{1}} \mathrm{~d} x_{2} \int_{0}^{1-x_{1}-x_{2}} \mathrm{~d} x_{3} \mathcal{Q}(\mathbf{x}) \mathcal{V}^{\mu}(\mathbf{x}) \\
& =\int_{0}^{1} \mathrm{~d} x_{1} \int_{0}^{1-x_{1}} \mathrm{~d} x_{2} \int_{0}^{1-x_{1}-x_{2}} \mathrm{~d} x_{3} \frac{1}{\Delta} \mathcal{V}^{\mu+1}(\mathbf{x}) \\
& \quad \times\left\{\mathcal{Q}(\mathbf{x})+\frac{1}{2(\mu+1)} \sum_{k=1}^{3} \frac{\partial}{\partial x_{k}}\left[\left(x_{k}+A_{k}\right) \mathcal{Q}(\mathbf{x})\right]\right\} \\
& \quad-\left.\int_{0}^{1} \mathrm{~d} x_{1} \int_{0}^{1-x_{1}} \mathrm{~d} x_{2} \frac{1}{\Delta} \frac{1}{2(\mu+1)}\left(1+\sum_{k=1}^{3} A_{k}\right) \mathcal{Q}(\mathbf{x}) \mathcal{V}^{\mu+1}(\mathbf{x})\right|_{x_{3}=1-x_{1}-x_{2}} \\
& \quad+\left.\int_{0}^{1} \mathrm{~d} x_{1} \int_{0}^{1-x_{1}} \mathrm{~d} x_{2} \frac{1}{\Delta} \frac{1}{2(\mu+1)} A_{3} \mathcal{Q}(\mathbf{x}) \mathcal{V}^{\mu+1}(\mathbf{x})\right|_{x_{3}=0} \\
& \quad+\left.\int_{0}^{1} \mathrm{~d} x_{1} \int_{0}^{1-x_{1}} \mathrm{~d} x_{3} \frac{1}{\Delta} \frac{1}{2(\mu+1)} A_{2} \mathcal{Q}(\mathbf{x}) \mathcal{V}^{\mu+1}(\mathbf{x})\right|_{x_{2}=0} \\
& \quad+\left.\int_{0}^{1} \mathrm{~d} x_{2} \int_{0}^{1-x_{2}} \mathrm{~d} x_{3} \frac{1}{\Delta} \frac{1}{2(\mu+1)} A_{1} \mathcal{Q}(\mathbf{x}) \mathcal{V}^{\mu+1}(\mathbf{x})\right|_{x_{1}=0} .
\end{aligned}
$$

This step can be applied iteratively until the power of $\mathcal{V}$ is as high as required, optimally $\mu=-n+\epsilon \rightarrow \epsilon$, where $n$ is a positive integer. In this way, the original integral is expressed in terms of a sum of different integrals, which possess better integration properties.

Although no general and compact-form solution of the BT algorithm is known for problems beyond the one-loop case, it is only natural to apply a one-loop BT relation to a sub-loop of a two-loop integral. In this way, the integral can be made smooth with respect to the Feynman parameters connected with the sub-loop to which the BT procedure is applied. Due to the size of the expressions and their divergency structure, it is usually better to apply this relation to the sub-loop with the highest number of internal lines.

Finally, the $\epsilon$ poles in each component $x_{i}$ of the vector $\mathbf{x}$ are extracted by the relation

$$
\begin{aligned}
& \int_{0}^{1} \mathrm{~d} x_{i} x_{i}^{-n+\epsilon} f(\mathbf{x}) \\
& =\int_{0}^{1} \mathrm{~d} x_{i} x_{i}^{-n+\epsilon}\left(f(\mathbf{x})-\sum_{k=0}^{n-1} x_{i}^{k} \frac{f^{(k)}(0)}{k !}\right)+\sum_{k=0}^{n-1} \frac{f^{(k)}(0)}{k !(k+1-n+\epsilon)},
\end{aligned}
$$

where $i=1, \ldots, n$. 


\subsection{Semi-numerical integration based on dispersion relations}

This method makes use of the fact that the one-loop self-energy can be written, with the help of a dispersion relation, as an integral over an expression that has the analytical form of a propagator

$$
\begin{aligned}
B_{0}\left(p^{2}, m_{1}^{2}, m_{2}^{2}\right) & =\int_{\left(m_{1}+m_{2}\right)^{2}}^{\infty} \mathrm{d} s \frac{\Delta B_{0}\left(s, m_{1}^{2}, m_{2}^{2}\right)}{s-p^{2}}, \\
\Delta B_{0}\left(s, m_{1}^{2}, m_{2}^{2}\right) & =\left(4 \pi \mu^{2}\right)^{4-D} \frac{\Gamma(D / 2-1)}{\Gamma(D-2)} \frac{\lambda^{(D-3) / 2}\left(s, m_{1}^{2}, m_{2}^{2}\right)}{s^{D / 2-1}},
\end{aligned}
$$

where $\lambda(x, y, z)=x^{2}+y^{2}+z^{2}-2(x y+y z+z x)$. If this one-loop self-energy is a sub-loop of a two-loop integral, the dispersion relation effectively reduces this integral to a one-loop integral with the additional integration over $s$, which is performed numerically [21].

Integrals with sub-loop triangles can be reduced to integrals with sub-loop self-energies by introducing Feynman parameters [26]. The integration over the Feynman parameters is also performed numerically. More details can be found in Ref. [7]. If the two-loop integrals contains ultraviolet, infrared or threshold divergences, they need to be subtracted before the numerical integration can be carried out. For our purposes, this is achieved by subtracting a term from the integrand that can be integrated analytically.

The reduction of integrals with irreducible numerators to a small set of master integrals is accomplished by using integration-by-parts and Lorentz-invariance identities. For complex diagrams with triangle sub-loops, the number of required relations can become very large, which is a limitation of this method. Therefore we do not use it to compute the complete result for the two-loop corrections to $\sin ^{2} \theta_{\mathrm{eff}}^{b \bar{b}}$, but only for cross checks of individual integrals and diagrams.

\subsection{Comparison of methods}

In this section, we compare our three methods, based on the top-quark mass expansion algorithm, the BT method, and the dispersion relations, where applicable. For the comparison, we use the following dimensionless input parameters: $M_{Z}=1, M_{W}=80 / 91$, $m_{t}=180 / 91$, and the scale for dimensional regularisation $\mu=e^{\gamma_{\mathrm{E}}}$. The large-mass expansion is performed down to $\mathcal{O}\left(m_{t}^{-12}\right)$. The expressions are normalised as they enter in $\sin ^{2} \theta_{\text {eff }}^{b \bar{b}}$, with the common prefactor $(\alpha / 4 \pi)^{2}$ factored out. For the comparison, we selected the set of diagrams corresponding to Fig. 1, where only $W$-boson propagators in Feynman gauge, but not Goldstone-boson exchange has been included. For light-fermion loops, results are shown for leptons in the sub-loops, summed over the three lepton families, and for diagram $(\mathrm{G})$ we chose the $l l \nu$ sub-loop, also summed over generations. Our results are presented in Table 1.

Where available, the results from the BT method and the method based on dispersion relations agree to all digit shown in the table. As mentioned above, no complete results for diagrams $(\mathrm{E})-(\mathrm{G})$ could be obtained with the dispersion relation method. 


\begin{tabular}{lllll}
\hline Diagram & Method & Result $\left[(\alpha / 4 \pi)^{2}\right]$ & & \\
\hline$(\mathrm{A})$ & semi-numerical & $+3.82775120 / \epsilon^{2}$ & $+3.88128795 / \epsilon$ & -19.1983330 \\
& $m_{t}$ expansion & $+3.82775120 / \epsilon^{2}$ & $+3.88 / \epsilon$ & -19.19 \\
\hline (B) & semi-numerical & $+3.82775120 / \epsilon^{2}$ & $-8.67823832 / \epsilon$ & +25.4468576 \\
& $m_{t}$ expansion & $+3.82775120 / \epsilon^{2}$ & $-8.68 / \epsilon$ & +25.45 \\
\hline$(\mathrm{C})$ & semi-numerical & $0 / \epsilon^{2}$ & $+0.90521614 / \epsilon$ & -0.60580110 \\
& $m_{t}$ expansion & $0 / \epsilon^{2}$ & $+0.905 / \epsilon$ & -0.61 \\
\hline$(\mathrm{D})$ & semi-numerical & $0 / \epsilon^{2}$ & $+1.55085212 / \epsilon$ & -4.50488822 \\
& $m_{t}$ expansion & $0 / \epsilon^{2}$ & $+1.55 / \epsilon$ & -4.50 \\
\hline$(\mathrm{E})$ & semi-numerical & $-2.30183413 / \epsilon^{2}$ & $+5.07108758 / \epsilon$ & +8.32594367 \\
& $m_{t}$ expansion & $-2.30183413 / \epsilon^{2}$ & $+5.07 / \epsilon$ & +8.33 \\
\hline$(\mathrm{F})$ & semi-numerical & $-2.80183413 / \epsilon^{2}$ & $+6.17261951 / \epsilon$ & -14.028 \\
& $m_{t}$ expansion & $-2.80183413 / \epsilon^{2}$ & $+6.17 / \epsilon$ & -14.03 \\
\hline$(\mathrm{G})$ & semi-numerical & $-1.80183413 / \epsilon^{2}$ & $+3.9695556(5) / \epsilon$ & -13.539 \\
& $m_{t}$ expansion & $-1.80183413 / \epsilon^{2}$ & $+3.97 / \epsilon$ & -13.54 \\
\hline
\end{tabular}

Table 1: Comparison of the top-quark mass expansion with semi-numerical integrations for selected diagrams of Fig. 1. For diagrams (A)-(D), the semi-numerical results for the BT and dispersion relation methods agree to all given digits, while for diagrams $(\mathrm{E})-(\mathrm{G})$ results are available only for the BT method.

There are clear advantages to the use of large- $m_{t}$ asymptotic expansions. No special treatment is required for the different types of divergences. Each large-mass pattern is associated with one expansion scheme, which is not sensitive to threshold divergences produced by the presence of small masses. In effect, the resulting programs are general and concise. As can be seen from Table 1, the obvious drawback of this method is the limited precision of the final results. However, it was observed that, with reasonable investment of computer time, the precision can be pushed to as high as required by the problem at hand.

The semi-numerical programs usually produce results of better precision. However, the iterative application of the BT relation should be kept at a minimum, as otherwise the precision can actually be lost. At least in our realization, the semi-numerical programs based on the BT and dispersion relation methods are not as general as the expansion technique. Special care has to be taken to deal with threshold-divergent cases, and the size of intermediate expressions can actually be much larger than what is observed during the use of the large-mass expansion. In the end, the time required for the analytic simplifications of these semi-numerical programs can be as large as the time required for performing a high-order large-mass expansion.

It should also be kept in mind that the algorithms for asymptotic expansions can be easily generalised to higher orders in perturbation theory, and were already applied for three-loop problems; see, for example, Refs. [27,28]. The application of the BT method 


\begin{tabular}{ll}
\hline Input parameter & Value \\
\hline$M_{W}$ & $(80.404 \pm 0.0030) \mathrm{GeV}$ \\
$M_{Z}$ & $(91.1876 \pm 0.0021) \mathrm{GeV}$ \\
$\Gamma_{Z}$ & $2.4952 \mathrm{GeV}$ \\
$m_{t}$ & $(172.5 \pm 2.3) \mathrm{GeV}$ \\
$m_{b}$ & $4.85 \mathrm{GeV}$ \\
$\Delta \alpha\left(M_{Z}\right)$ & $0.05907 \pm 0.00036$ \\
$\alpha_{s}\left(M_{Z}\right)$ & $0.119 \pm 0.002$ \\
$G_{\mu}$ & $1.16637 \times 10^{-5} \mathrm{GeV}^{-2}$ \\
\hline
\end{tabular}

Table 2: Experimental input parameters used in the numerical evaluation, from Refs. [31, $32]$.

in such cases is more complicated, and no physical results are known at this moment.

\section{Results}

The computational methods described in the previous section were implemented in automatised computer codes, to be able to handle the large-size expressions at intermediate stages. The analytic algorithm for the BT reduction was written in FORM [29] and Mathematica. The code for the large-top-quark-mass expansion was also implemented in FORM. When necessary, the reduction of two-loop propagators with higher powers of numerators and denominators, which inevitably appear for higher orders of the asymptotic expansion, was performed with the program IdSolver [4,25]. For the problem at hand, it had to create and solve a set of several thousands of equations, which it can achieve with very little computing time. For the numerical integrations, we developed a fast code written in $\mathrm{C}$ with the help of the CuBA library [30].

The results presented here were tested at different levels. We checked the finiteness and gauge invariance of the two-loop contributions to $\sin ^{2} \theta_{\text {eff }}^{b \bar{b}}$ analytically. The numerical results for the new diagrams were tested with two different methods, as presented in the previous section. In addition, full evaluation was performed independently by two groups within our collaboration.

In the following, we show our numerical results for the input parameters listed in Table 2. For the sake of easy comparison, we use the same parameters as in previous publications on $\sin ^{2} \theta_{\text {eff }}^{\text {lept }}$ [7], which is justified by the fact that the changes of measured values are insignificant for this presentation. It is important to note that the experimental values for the $W$ - and $Z$-boson masses correspond to a Breit-Wigner parametrisation with a running width and have to be translated to the pole-mass scheme used in the loop calculations [14]. In effect, this translation results in a downward shift [33] of $M_{Z}$ and $M_{W}$ by about 34 and $28 \mathrm{MeV}$, respectively. The non-zero mass of the bottom quark was retained in the $\mathcal{O}(\alpha)$ contribution, but neglected in the two-loop part. The complete fermionic two-loop contribution to $\sin ^{2} \theta_{\text {eff }}^{b b}$ is shown in Fig. 2, for various levels of the 


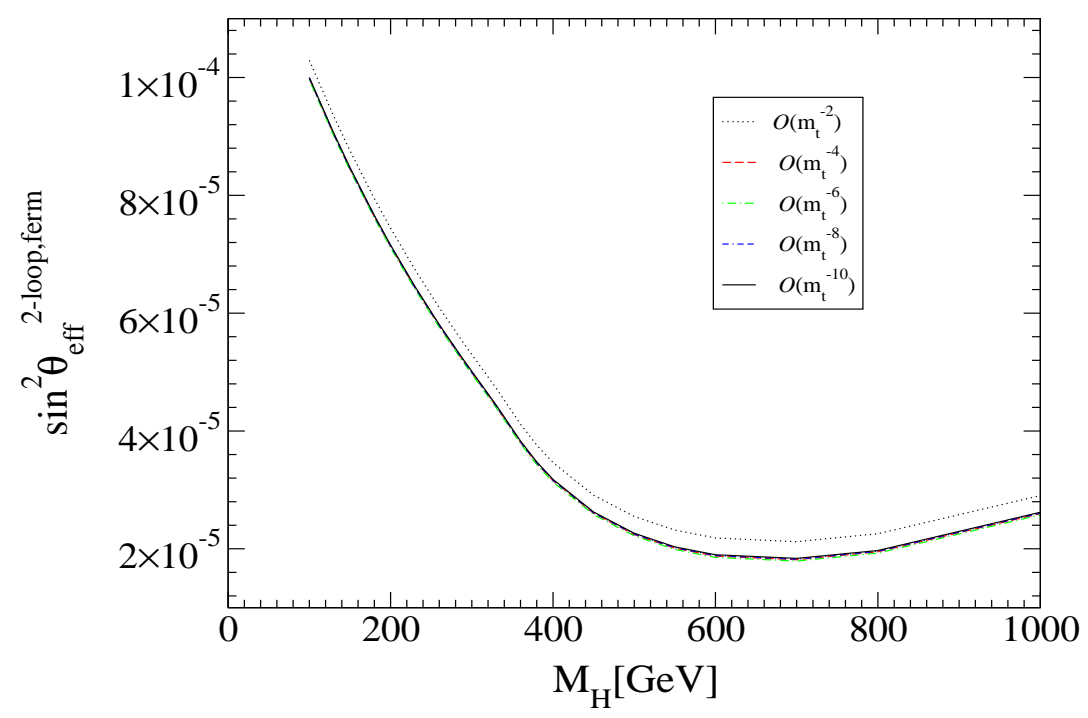

Figure 2: Two-loop fermionic contribution to $\sin ^{2} \theta_{\text {eff }}^{b \bar{b}}$, with the $W$-boson mass evaluated from the Standard Model and the other input parameters taken from Table 2.

\begin{tabular}{lll}
\hline$M_{H}$ & $\mathcal{O}(\alpha)$ & $\mathcal{O}\left(\alpha_{4}^{2}\right)_{\text {ferm }}$ \\
{$[\mathrm{GeV}]$} & {$\left[10^{-4}\right]$} & {$\left[10^{-4}\right]$} \\
\hline 100 & 104.77 & 1.00 \\
200 & 100.15 & 0.71 \\
400 & 94.397 & 0.32 \\
600 & 90.666 & 0.19 \\
1000 & 85.748 & 0.26 \\
\hline
\end{tabular}

\begin{tabular}{lll}
\hline$M_{H}$ & $\mathcal{O}(\alpha)$ & $\mathcal{O}\left(\alpha^{2}\right)_{\text {ferm }}$ \\
{$[\mathrm{GeV}]$} & {$\left[10^{-4}\right]$} & {$\left[10^{-4}\right]$} \\
\hline 100 & 105.03 & 0.98 \\
200 & 100.74 & 0.67 \\
400 & 95.354 & 0.24 \\
600 & 91.847 & 0.10 \\
1000 & 87.196 & 0.16 \\
\hline
\end{tabular}

Table 3: Contributions to $\sin ^{2} \theta_{\text {eff }}^{b \bar{b}}$, with the $W$-boson mass evaluated from the Standard Model (left) or fixed (right).

large-top-quark-mass expansion. For the plot, $G_{\mu}$ is used as an input parameter, and $M_{W}$ is calculated from it according to Eq. (3). As can be seen from the figure, the asymptotic expansion is converging robustly over the entire range of relevant Higgs-boson mass values. The relative error estimated for the expansion up to $\mathcal{O}\left(m_{t}^{-10}\right)$ is $10^{-5}$ and thus more than sufficient for our purposes. The numerical values for selected values of the Higgs-boson mass are shown in Table 3. The left table uses $G_{\mu}$ as an input via Eq. (3), while a fixed mass $M_{W}=80.404 \mathrm{GeV}$ is used for the right table. For small Higgs-boson mass, the new correction is relatively large, about $10^{-4}$. For larger values of $M_{H}$, it can be up to one order of magnitude smaller, about $10^{-5}$. Following earlier publications on two-loop electroweak corrections, we express our results in terms of fitting formulas. The form factor $\Delta \kappa_{b \bar{b}}^{\left(\alpha^{2} \text {,ferm }\right)}$, which contains the fermionic two-loop electroweak corrections to 
$\sin ^{2} \theta_{\text {eff }}^{b \bar{b}}$ according to Eq. (2), can be approximated as

$$
\begin{aligned}
\Delta \kappa_{b \bar{b}}^{\left(\alpha^{2}, \text { ferm }\right)}= & \Delta \alpha \Delta \kappa_{b \bar{b}}^{(\alpha)}+\Delta \kappa_{b \bar{b}, \text { rem }}^{\left(\alpha^{2}, \text { ferm }\right)} \\
\Delta \kappa_{b \bar{b}, \text { rem }}^{\left(\alpha^{2} \text {,ferm }\right)}= & k_{0}+k_{1} L_{H}+k_{2} L_{H}^{2}+k_{3} L_{H}^{4}+k_{4}\left(\Delta_{H}^{2}-1\right)+k_{5} \Delta_{t} \\
& +k_{6} \Delta_{t}^{2}+k_{7} \Delta_{t} L_{H}+k_{8} \Delta_{W}+k_{9} \Delta_{W} \Delta_{t}+k_{10} \Delta_{Z}
\end{aligned}
$$

where $\Delta \kappa_{b \bar{b}}^{(\alpha)}$ is the one-loop result, and

$$
\begin{aligned}
L_{H} & =\ln \frac{M_{H}}{100 \mathrm{GeV}}, & \Delta_{H} & =\frac{M_{H}}{100 \mathrm{GeV}}, \\
\Delta_{Z} & =\frac{M_{Z}}{91.1876 \mathrm{GeV}}-1, & \Delta_{W} & =\frac{M_{W}}{80.404 \mathrm{GeV}}-1 .
\end{aligned}
$$

Fitting this formula to the exact result, we obtain

$$
\begin{array}{lll}
k_{0}=-2.666 \times 10^{-3}, & k_{1}=-5.92 \times 10^{-5}, & k_{2}=-3.29 \times 10^{-6}, \\
k_{3}=3.49 \times 10^{-6}, & k_{4}=2.83 \times 10^{-6}, & k_{5}=-5.34 \times 10^{-3}, \\
k_{6}=-2.10 \times 10^{-3}, & k_{7}=-2.19 \times 10^{-4}, & k_{8}=-6.31 \times 10^{-2}, \\
k_{9}=-1.26 \times 10^{-1}, & k_{10}=6.47 \times 10^{-2} . &
\end{array}
$$

This parametrisation reproduces the exact calculation with maximal and average deviations of $1.4 \times 10^{-5}$ and $5 \times 10^{-6}$, respectively, as long as the input parameters stay within their $2 \sigma$ ranges of the experimental errors quoted in Table 2 and the Higgs-boson mass is in the range $10 \mathrm{GeV} \leq M_{H} \leq 1 \mathrm{TeV}$. If the top-quark mass and the $W$-boson mass vary within $4 \sigma$ ranges, the formula is still accurate to $2.1 \times 10^{-5}$.

We also present a simple parametrisation for the currently best prediction for $\sin ^{2} \theta_{\text {eff }}^{b \bar{b}}$, including all known corrections to $\Delta \kappa_{b \bar{b}}$ and $\Delta r$ (for the calculation of $M_{W}$ from $G_{\mu}$ see Refs. [2,34]). For $\Delta \kappa_{b \bar{b}}$, in addition to the one-loop and fermionic two-loop electroweak corrections, we include QCD corrections of $\mathcal{O}\left(\alpha \alpha_{s}\right)$ [35] and $\mathcal{O}\left(\alpha \alpha_{s}^{2}\right)[27,36]$ to the oneloop contribution, as well as universal corrections for large top-quark mass, of $\mathcal{O}\left(\alpha^{2} \alpha_{s} m_{t}^{4}\right)$ and $\mathcal{O}\left(\alpha^{3} m_{t}^{6}\right)$ [28]. Moreover, leading four-loop QCD correction to the $\rho$ parameter, which arise from top- and bottom-quark loops, are taken into account [37]. We use the parametrisation

$$
\begin{aligned}
\sin ^{2} \theta_{\mathrm{eff}}^{b \bar{b}}= & s_{0}+d_{1} L_{H}+d_{2} L_{H}^{2}+d_{3} L_{H}^{4}+d_{4}\left(\Delta_{H}^{2}-1\right)+d_{5} \Delta_{\alpha} \\
& +d_{6} \Delta_{t}+d_{7} \Delta_{t}^{2}+d_{8} \Delta_{t}\left(\Delta_{H}-1\right)+d_{9} \Delta_{\alpha_{s}}+d_{10} \Delta_{Z}
\end{aligned}
$$

with

$$
\Delta_{\alpha}=\frac{\Delta \alpha\left(M_{Z}\right)}{0.05907}-1, \quad \Delta_{\alpha_{s}}=\frac{\alpha_{s}\left(M_{Z}\right)}{0.117}-1
$$

The best-fit numerical values for the coefficients are

$$
\begin{array}{llrl}
s_{0}=2.327580 \times 10^{-1}, & d_{1}=4.749 \times 10^{-4}, & d_{2}=2.03 \times 10^{-5} \\
d_{3}=3.94 \times 10^{-6}, & d_{4}=-1.84 \times 10^{-6}, & d_{5}=2.08 \times 10^{-2} \\
d_{6}=-9.93 \times 10^{-4}, & d_{7}=7.08 \times 10^{-5}, & d_{8}=-7.61 \times 10^{-6} \\
d_{9}=4.03 \times 10^{-4}, & d_{10}=6.61 \times 10^{-1} . &
\end{array}
$$


This parametrisation approximates the full result with maximal and average deviations of $4.3 \times 10^{-6}$ and $1.3 \times 10^{-6}$, respectively, for $10 \mathrm{GeV} \leq M_{H} \leq 1 \mathrm{TeV}$ and the other input parameters in their $2 \sigma$ ranges.

\section{Conclusions}

In this paper, the calculation of the two-loop electroweak fermionic corrections to the effective weak-mixing angle for the $Z b \bar{b}$ vertex, $\sin ^{2} \theta_{\text {eff }}^{b \bar{b}}$, was presented. Such an accurate theoretical prediction for $\sin ^{2} \theta_{\text {eff }}^{b \bar{b}}$ is necessary for the interpretation of the bottom-quark asymmetry measurements at the $Z$-boson pole. Compared to the previously known corrections to $\sin ^{2} \theta_{\text {eff }}^{b \bar{b}}$, the new electroweak two-loop result turns out to be sizable, of order $\mathcal{O}\left(10^{-4}\right)$ for a Higgs-boson mass near $100 \mathrm{GeV}$.

The calculation was performed by using methods that had been used earlier for the computation of the leptonic effective weak-mixing angle, as well as a newly developed code based on the BT algorithm. The results of the different methods were checked against each other.

Although we did not perform a detailed analysis of the error from unknown high-order corrections, in particular the missing bosonic two-loop corrections and terms of order $\mathcal{O}\left(\alpha^{2} \alpha_{s}\right)$, we expect those to be of similar order as for the leptonic effective weak-mixing angle. The main difference between the leptonic and bottom-quark effective weak-mixing angles are the vertex diagrams with internal $W$-boson and top-quark propagators. While leading to numerical differences between $\sin ^{2} \theta_{\text {eff }}^{\text {lept }}$ and $\sin ^{2} \theta_{\text {eff }}^{b \vec{b}}$, these diagrams do not introduce special enhancement or suppression factors. Therefore, we expect the theoretical uncertainty to our result for $\sin ^{2} \theta_{\text {eff }}^{b \bar{b}}$ to be about $5 \times 10^{-5}$, similar to Ref. [7].

\section{Acknowledgements}

The work of M.A. and B.A.K. was supported in part by the German Research Foundation (DFG) through Grant No. KN 365/3-1 and through the Collaborative Research Centre No. 676 Particles, Strings and the Early Universe - the structure of Matter and Space Time. The work of M.C. was supported in part by the Sofja Kovalevskaja Award of the Alexander von Humboldt Foundation and by the ToK Program ALGOTOOLS (MTKDCD-2004-014319). A.F. is grateful for warm hospitality at Argonne National Laboratory and the Enrico Fermi Institute of the University of Chicago, where part of his work on this project was performed.

\section{References}

[1] A. Sirlin, Phys. Rev. D 22 (1980) 971.

[2] M. Awramik, M. Czakon, A. Freitas, G. Weiglein, Phys. Rev. D 69 (2004) 053006, arXiv:hep-ph/0311148. 
[3] M. Awramik, M. Czakon, A. Freitas, G. Weiglein, Phys. Rev. Lett. 93 (2004) 201805, arXiv:hep-ph/0407317;

M. Awramik, M. Czakon, A. Freitas, G. Weiglein, in: Proceedings of the International Conference on Linear Colliders (LCWS 04), Paris, France, 19-24 April 2004, arXiv:hep-ph/0409142;

A. Freitas, M. Awramik, M. Czakon, in: Proceedings of the 2005 International Linear Collider Workshop (LCWS 05), Stanford, California, 18-22 March 2005, p. 0610, arXiv:hep-ph/0507159.

[4] M. Awramik, M. Czakon, A. Freitas, G. Weiglein, Nucl. Phys. B (Proc. Suppl.) 135 (2004) 119, arXiv:hep-ph/0408207.

[5] W. Hollik, U. Meier, S. Uccirati, Nucl. Phys. B 731 (2005) 213, arXiv:hep$\mathrm{ph} / 0507158$

W. Hollik, U. Meier, S. Uccirati, Phys. Lett. B 632 (2006) 680, arXiv:hep-ph/0509302; W. Hollik, U. Meier, S. Uccirati, Nucl. Phys. B 765 (2007) 154, arXiv:hep$\mathrm{ph} / 0610312$.

[6] M. Awramik, M. Czakon, A. Freitas, Phys. Lett. B 642 (2006) 563, arXiv:hep$\mathrm{ph} / 0605339$

M. Czakon, M. Awramik, A. Freitas, Nucl. Phys. B (Proc. Suppl.) 157 (2006) 58, arXiv:hep-ph/0602029.

[7] M. Awramik, M. Czakon, A. Freitas, JHEP 0611 (2006) 048, arXiv:hep-ph/0608099.

[8] ALEPH, DELPHI, L3 and OPAL Collaborations, S. Schael, et al., Phys. Rept. 427 (2006) 257, arXiv:hep-ex/0509008.

[9] R. Hawkings, K. Mönig, Eur. Phys. J. direct C 1 (1999) 8, arXiv:hep-ex/9910022.

[10] R. Barbieri, M. Beccaria, P. Ciafaloni, G. Curci, A. Vicere, Phys. Lett. B 288 (1992) 95, arXiv:hep-ph/9205238;

R. Barbieri, M. Beccaria, P. Ciafaloni, G. Curci, A. Vicere, Phys. Lett. B 312 (1993) 511, Erratum;

R. Barbieri, M. Beccaria, P. Ciafaloni, G. Curci, A. Vicere, Nucl. Phys. B 409 (1993) 105 ;

J. Fleischer, O.V. Tarasov, F. Jegerlehner, Phys. Lett. B 319 (1993) 249;

J. Fleischer, O.V. Tarasov, F. Jegerlehner, Phys. Rev. D 51 (1995) 3820;

A. Denner, W. Hollik, B. Lampe, Z. Phys. C 60 (1993) 193, arXiv:hep-ph/9305273;

J. Fleischer, F. Jegerlehner, M. Tentyukov, O.L. Veretin, Phys. Lett. B 459 (1999) 625, arXiv:hep-ph/9904256.

[11] D. Bardin, et al., Report No. CERN-TH.6443/92, arXiv:hep-ph/9412201;

D. Bardin, M. Bilenky, P. Christova, M. Jack, L. Kalinovskaya, A. Olchevski, S. Riemann, T. Riemann, Comput. Phys. Commun. 133 (2001) 229, arXiv:hep-ph/9908433; A.B. Arbuzov, M. Awramik, M. Czakon, A. Freitas, M.W. Grünewald, K. Mönig, 
S. Riemann, T. Riemann, Comput. Phys. Commun. 174 (2006) 728, arXiv:hep$\mathrm{ph} / 0507146$.

[12] H. Flächer, M. Goebel, J. Haller, A. Höcker, K. Mönig and J. Stelzer, arXiv:0811.0009 [hep-ph].

[13] A. Freitas, K. Mönig, Eur. Phys. J. C 40 (2005) 493, arXiv:hep-ph/0411304.

[14] A. Freitas, W. Hollik, W. Walter, G. Weiglein, Nucl. Phys. B 632 (2002) 189, arXiv:hep-ph/0202131;

A. Freitas, W. Hollik, W. Walter, G. Weiglein, Nucl. Phys. B 666 (2003) 305, Erratum.

[15] M. Butenschön, F. Fugel, B.A. Kniehl, Phys. Rev. Lett. 98 (2007) 071602, arXiv:hep$\mathrm{ph} / 0612184$

M. Butenschön, F. Fugel, B.A. Kniehl, Nucl. Phys. B 772 (2007) 25, arXiv:hepph/0702215.

[16] V.A. Smirnov, Evaluating Feynman Integrals, Springer Tracts Mod. Phys. 211 (2004) 1

V.A. Smirnov, Applied asymptotic expansions in momenta and masses, Springer, Berlin, Germany, 2002.

[17] F.V. Tkachov, Nucl. Instrum. Meth. A 389 (1997) 309, arXiv:hep-ph/9609429.

[18] D.Yu. Bardin, L.V. Kalinovskaya, F.V. Tkachov, in: Proceedings of the 15th International Workshop on High-Energy Physics and Quantum Field Theory (QFTHEP 2000), Tver, Russia, 14-20 September 2000, arXiv:hep-ph/0012209;

G. Passarino, Nucl. Phys. B 619 (2001) 257, arXiv:hep-ph/0108252;

A. Ferroglia, M. Passera, G. Passarino, S. Uccirati, Nucl. Phys. B 650 (2003) 162, arXiv:hep-ph/0209219.

[19] A. Ferroglia, M. Passera, G. Passarino, S. Uccirati, Nucl. Phys. B 680 (2004) 199, arXiv:hep-ph/0311186;

S. Actis, A. Ferroglia, G. Passarino, M. Passera, S. Uccirati, Nucl. Phys. B 703 (2004)

3, arXiv:hep-ph/0402132;

G. Passarino, S. Uccirati, Nucl. Phys. B 747 (2006) 113, arXiv:hep-ph/0603121.

[20] G. Passarino, C. Sturm, S. Uccirati, Phys. Lett. B 655 (2007) 298, arXiv:0707.1401 [hep-ph].

[21] S. Bauberger, F.A. Berends, M. Böhm, M. Buza, Nucl. Phys. B 434 (1995) 383, arXiv:hep-ph/9409388;

B.A. Kniehl, Acta Phys. Polon. B 27 (1996) 3631, arXiv:hep-ph/9607255.

[22] S. Laporta, Int. J. Mod. Phys. A 15 (2000) 5087, arXiv:hep-ph/0102033. 
[23] K.G. Chetyrkin, F.V. Tkachov, Nucl. Phys. B 192 (1981) 159.

[24] T. Gehrmann, E. Remiddi, Nucl. Phys. B 580 (2000) 485, arXiv:hep-ph/9912329.

[25] M. Czakon, DiaGen/IdSolver (unpublished).

[26] A. Ghinculov, J.J. van der Bij, Nucl. Phys. B 436 (1995) 30, arXiv:hep-ph/9405418.

[27] K.G. Chetyrkin, J.H. Kühn, M. Steinhauser, Phys. Rev. Lett. 75 (1995) 3394, arXiv:hep-ph/9504413.

[28] M. Faisst, J.H. Kühn, T. Seidensticker, O. Veretin, Nucl. Phys. B 665 (2003) 649, arXiv:hep-ph/0302275.

[29] J.A.M. Vermaseren, Report No. NIKHEF-00-032, arXiv:math-ph/0010025.

[30] T. Hahn, Comput. Phys. Commun. 168 (2005) 78, arXiv:hep-ph/0404043.

[31] LEP Electroweak Working Group, D. Abbaneo, et al., http://lepewwg.web.cern.ch/LEPEWWG/.

[32] Particle Data Group, S. Eidelman, et al., Phys. Lett. B 592 (2004) 1; also 2005 partial update for edition 2006, available on http://pdg.lbl.gov.

[33] D.Yu. Bardin, A. Leike, T. Riemann, M. Sachwitz, Phys. Lett. B 206 (1988) 546.

[34] A. Freitas, W. Hollik, W. Walter, G. Weiglein, Phys. Lett. B 495 (2000) 338, arXiv:hep-ph/0007091;

A. Freitas, W. Hollik, W. Walter, G. Weiglein, Phys. Lett. B 570 (2003) 260, Erratum;

M. Awramik, M. Czakon, Phys. Rev. Lett. 89 (2002) 241801, arXiv:hep-ph/0208113;

M. Awramik, M. Czakon, A. Onishchenko, O. Veretin, Phys. Rev. D 68 (2003) 053004, arXiv:hep-ph/0209084;

A. Onishchenko, O. Veretin, Phys. Lett. B 551 (2003) 111, arXiv:hep-ph/0209010;

M. Awramik, M. Czakon, Phys. Lett. B 568 (2003) 48, arXiv:hep-ph/0305248.

[35] A. Djouadi, C. Verzegnassi, Phys. Lett. B 195 (1987) 265;

A. Djouadi, Nuovo Cim. A 100 (1988) 357;

B.A. Kniehl, Nucl. Phys. B 347 (1990) 86;

F. Halzen, B.A. Kniehl, Nucl. Phys. B 353 (1991) 567;

B.A. Kniehl, A. Sirlin, Nucl. Phys. B 371 (1992) 141;

B.A. Kniehl, A. Sirlin, Phys. Rev. D 47 (1993) 883;

A. Djouadi, P. Gambino, Phys. Rev. D 49 (1994) 3499, arXiv:hep-ph/9309298;

A. Djouadi, P. Gambino, Phys. Rev. D 53 (1996) 4111, Erratum. 
[36] L. Avdeev, J. Fleischer, S. Mikhailov, O. Tarasov, Phys. Lett. B 336 (1994) 560, arXiv:hep-ph/9406363;

L. Avdeev, J. Fleischer, S. Mikhailov, O. Tarasov, Phys. Lett. B 349 (1994) 597, Erratum;

K.G. Chetyrkin, J.H. Kühn, M. Steinhauser, Phys. Lett. B 351 (1995) 331, arXiv:hep$\mathrm{ph} / 9502291$

Y. Schröder, M. Steinhauser, Phys. Lett. B 622 (2005) 124, arXiv:hep-ph/0504055.

[37] K.G. Chetyrkin, M. Faisst, J.H. Kühn, P. Maierhofer, C. Sturm, Phys. Rev. Lett. 97 (2006) 102003, arXiv:hep-ph/0605201;

R. Boughezal, M. Czakon, Nucl. Phys. B 755 (2006) 221, arXiv:hep-ph/0606232. 